\title{
Comment on "Energy levels, oscillator strengths, and transition probabilities for sulfur-like scandium, Sc VI" by El-Maaref et al. [Indian J. Phys. 911029 (2017)]
}

\author{
K M Aggarwal* (1) \\ Astrophysics Research Centre, School of Mathematics and Physics, Queen's University Belfast, Belfast, Northern Ireland BT7 1NN, UK
}

Received: 13 May 2019 / Accepted: 29 November 2019 / Published online: 10 June 2020

\begin{abstract}
In this comment, through our independent calculations, we assess that the recently reported results of El-Maaref et al. [Indian J. Phys. 911029 (2017)] for energy levels, oscillator strengths, radiative rates, and lifetimes are inaccurate and unreliable for several levels and transitions of S-like Sc VI.
\end{abstract}

Keywords: Energy levels; Oscillator strengths; Lifetimes; S-like scandium Sc VI

PACS Nos.: $32.70 . C s ; 95.30 . \mathrm{Ky}$

\section{Introduction}

Scandium (Sc) belongs to the group of rare earth elements and hence atomic data for its ions are useful for the studies of fusion plasmas [1]. It is also an important element for the studies of astrophysical plasmas, as discussed by Pryce [2], and is a key element for understanding Am stars, as demonstrated by LeBlanc and Alecian [3]. For this reason, several workers have reported atomic data for its ions-see for example, the early extensive work of Massacrier and Artru [4], who have reported atomic data for several ions of this element, i.e. Sc III to Sc XXI.

In one of the recent papers, El-Maaref et al. [5] have reported results for energy levels, oscillator strengths $(f$ values), radiative rates ( $A$-values), and lifetimes $(\tau)$ for one of the important ions, namely S-like Sc VI. For the calculations, they have adopted two independent atomic structure codes, namely the configuration interaction version 3 (CIV3 [6]) and the Los Alamos National Laboratory (LANL) code, which is mainly based on the relativistic Hartree-Fock code of Cowan [7]. Based on these two calculations, and comparisons made with the existing theoretical and experimental results, they have assessed their data to be accurate and useful for applications. However,

*Corresponding author, E-mail: K.Aggarwal@qub.ac.uk we strongly disagree with their calculations and conclusions, as discussed below.

For most levels, the differences between the CIV3 and LANL energies are $\sim 2 \%$ (or $\sim 8000 \mathrm{~cm}^{-1}$ in absolute values), but up to $5 \%$ for a few, such as of $3 \mathrm{p}^{4}{ }^{3} \mathrm{P}_{1,2}$ - see table 2 of [5]. Since measurements of energies have also been made for some levels of Sc VI, these values have been compiled and assessed by the NIST (National Institute of Standards and Technology) team, and their recommended results are freely available at their website: https://physics.nist.gov/PhysRefData/ASD/

levels_form.html. The measured energies are (generally) considered to be more accurate and, therefore, the aim of most calculations is to match their results with those. Therefore, as expected, El-Maaref et al. [5] have also made comparisons between their calculated energies and those listed by NIST. For lower levels, such as 1-9 (see table 2 of [5]), the CIV3 results are comparatively more accurate, but for the higher ones, such as 48-55, the LANL energies are in (much) better agreement with those of NIST. Therefore, there is no consistency.

Unfortunately, experimental energies are not available for all the levels calculated by El-Maaref et al. [5], and neither are the theoretical ones, as may be noted from their table 2. However, it is not strictly true, because as stated earlier, Massacrier and Artru [4] have considered much larger number of levels, i.e. 1889, more than an order of magnitude than those calculated by El-Maaref et al., i.e. 
only 160 . They could not make comparisons with the earlier work as perhaps they were unaware of it. Anyway, it is not our (great) concern at present about the number of levels or their accuracy. However, based on the comparisons made by El-Maaref et al. or the ones briefly discussed by us above, we can state with confidence that there is scope for improvement in the calculated energies for the levels of Sc VI. But it may also be fair to state that there are no (large) discrepancies in their calculated energies. Therefore, we do not elaborate much on the energy levels and rather focus on larger discrepancies seen for other parameters, i.e. $f$ - and $A$-values, and $\tau$ in their tables 3 and 4 , respectively.

For the $f$-values (or related $A$-values in $\mathrm{s}^{-1}$, because $\mathrm{f}_{i j}=$ $1.49 \times 10^{-16} \lambda_{j i}^{2}\left(\omega_{j} / \omega_{i}\right) A_{j i}$, where $\lambda$ is the transition wavelength in $\AA$ and $\omega$ is the statistical weight), discrepancies between the calculations of El-Maaref et al. [5] and those of Froese Fischer et al. [8] with the multi-configuration Hartree-Fock (MCHF) method are up to three orders of magnitude for several transitions, see for example 33, 34 and 75 in their table 3 . There are a few more calculations by other workers (see [5] for references), but we are not considering those, because the ones by Froese Fischer et al. are perhaps the most accurate available to date. This is because they have considered a very (very) large configuration interaction $(\mathrm{CI})$, which is very important for moderately heavy ions of Sc. Similarly, differences between the two sets of $\tau$ values are up to three orders of magnitude, as may be noted for levels 35-37 in table 4 of [5]. Since the results of El-Maaref et al. are more recent, these are also 'expected' to be more accurate. Unfortunately that is not the case. More importantly, since these are the only two calculations available for $\tau$ for the levels of Sc VI, such large differences cannot be ignored but rather need to be explained, resolved and understood, which has not been done by El-Maaref et al. Therefore, through our independent calculations, we assess which data are more accurate and why.

\section{Calculations}

For the determination of energy levels, radiative rates, oscillator strengths, and lifetimes, we have adopted the General-purpose Relativistic Atomic Structure Package (GRASP). The original code, developed by Grant et al. [9], has undergone numerous modifications and improvements by many workers in the past, and as a result of it several published versions are available in the literature, but the one employed by us is known as GRASP0 and is currently hosted at the website http://amdpp.phys.strath.ac. uk/UK_APAP/codes.html. This is a fully relativistic code based on the $j j$ coupling scheme, although the two-body relativistic operators included in this (but not in CIV3) are not too important for a moderately heavy ion Sc VI. Therefore, differences (if any) in energies (or other related parameters) with other calculations will not be because of this, but for other reasons. Finally, in the calculations, we have preferred the choice of extended average level (EAL) in which a weighted (proportional to $2 j+1$ ) trace of the Hamiltonian matrix is minimised. This option produces a compromise set of orbitals describing closely lying states with moderate accuracy, and results obtained with other options, such as average level (AL), are comparable for all levels.

El-Maaref et al. [5] have considered 160 levels belonging to 8 configurations, namely $3 s^{2} 3 p^{4}, 3 s 3 p^{5}$, $3 s^{2} 3 p^{3} 3 d, 3 s^{2} 3 p^{3} 4 s / 4 p / 4 d$, and $3 s^{2} 3 p^{3} 5 s / 5 p$. In the calculations they have included limited CI, mainly among these 8 configurations. We have performed several calculations, gradually increasing the number of orbitals and configurations, and subsequently the CI. Our final calculations include 4498 levels, which belong to 41 configurations, namely $3 s^{2} 3 p^{4}, 3 s 3 p^{5}, 3 p^{6}, 3 s^{2} 3 p^{3} 3 d, 3 s 3 p^{4} 3 d, 3 p^{5} 3 d$, $3 \mathrm{~s}^{2} 3 \mathrm{p}^{2} 3 \mathrm{~d}^{2}, \quad 3 \mathrm{~s} 3 \mathrm{p}^{3} 3 \mathrm{~d}^{2}, \quad 3 \mathrm{~s}^{2} 3 \mathrm{p}^{3} 4 \ell, \quad 3 \mathrm{~s} 3 \mathrm{p}^{4} 4 \ell, \quad 3 \mathrm{~s}^{2} 3 \mathrm{p}^{2} 3 \mathrm{~d} 4 \ell$, $3 \mathrm{~s}^{2} 3 \mathrm{p} 3 \mathrm{~d}^{3}, 3 \mathrm{~s} 3 \mathrm{p}^{3} 3 \mathrm{~d} 4 \ell, 3 \mathrm{p}^{4} 3 \mathrm{~d}^{2}, 3 \mathrm{p}^{5} 4 \ell, 3 \mathrm{~s}^{2} \mathrm{p}^{2} 3 \mathrm{~d}^{3}, 3 \mathrm{~s}^{2} 3 \mathrm{p}^{3} 5 \ell$, and $3 \mathrm{~s}^{2} 3 \mathrm{p}^{3} 6 \ell(\ell \leq \mathrm{g})$. Inclusion of such a large $\mathrm{CI}$ is necessary for two reasons: (i) Sc VI is only moderately heavy and, more importantly, (ii) levels of these configurations intermix - see for example table 5 of Massacrier and Artru [4]. El-Maaref et al. have ignored many of the important configurations, such as $3 \mathrm{~s} 3 \mathrm{p}^{4} 3 \mathrm{~d}$ whose 56 levels appear early on, well before those of $3 \mathrm{p}^{3} 4 \ell$. Another example is $3 p^{6}$, which generates only one level but intermixes with those of $3 \mathrm{p}^{3} 3 \mathrm{~d}$. El-Maaref et al. have mostly included those configurations whose energy levels appear on the NIST website. Inclusion of these additional configurations not only improves the accuracy of the calculated energies but also (significantly) affects the further determinations of $\tau$, because contributions arising from these missing levels are also accounted for. Conversely, the 160 levels considered by them are not the lowest. To calculate lifetime for the highest level of their calculations, i.e. $3 \mathrm{p}^{3} 5 \mathrm{p}^{1} \mathrm{~S}_{0}$, one needs to consider at least 524 levels!

\section{Oscillator strengths}

As stated in Sect. 1, our focus is on the results for $f$ - (or $A$-) values. For weaker transitions (say with $f<0.1$ ), differences among different calculations may sometimes be considerably large, particularly for those with $f<10^{-3}$. This is because their resultant matrices are highly sensitive to the small mixing coefficients from different levels and 
configurations. However, comparatively stronger transitions are generally immune to the additive or cancellation effects of the coefficients, because of their larger magnitudes. Therefore, it is much easier to establish the (in)accuracy of a calculation for such transitions by comparing their $f$-values.

In Table 1 we list a few transitions which are comparatively strong(er) with $f$-values from the CIV3 calculations of El-Maaref et al. [5], Froese Fischer et al. [8] with MCHF, and our own work with GRASP. These representative transitions are sufficient to highlight the similarity (and or the discrepancies) among different calculations. For all transitions listed here (and many more-see table A of [10]) there is a satisfactory agreement between the MCHF and GRASP results, but the CIV $3 f$-values of El-Maaref et al. differ by up to three orders of magnitude. Clearly, the results of El-Maaref et al. are neither correct nor reliable.

\section{Lifetimes}

The lifetime of a level is determined as $\tau(\mathrm{s})=1.0 / \Sigma_{i} \mathrm{~A}_{j i}$ where the summation runs over all types of transitions, although the electric dipole (E1) ones are normally the most dominant in magnitude, and hence more important. Nevertheless, we have included contributions from other types as well, namely magnetic dipole (M1), electric quadrupole (E2), and magnetic quadrupole (M2), which have been ignored by El-Maaref et al. [5], but have also been considered by Froese Fischer et al. [8], as expected.

In Table 2 we list only those levels for which differences between the CIV3 and MCHF values of $\tau$ are very large, i.e. up to three orders of magnitude. Furthermore, for brevity, only the lowest few levels are considered here.

Table 1 Comparison of oscillator strengths ( $f$-values) for some transitions of Sc VI. $\mathrm{a} \pm \mathrm{b} \equiv \mathrm{a} \times 10^{ \pm b}$

\begin{tabular}{lllll}
\hline Lower level & Upper level & CIV3 & MCHF & GRASP \\
\hline $3 \mathrm{p}^{4}{ }^{3} \mathrm{P}_{2}$ & $3 \mathrm{p}^{3}\left({ }^{2} \mathrm{P}\right) 3 \mathrm{~d}{ }^{3} \mathrm{P}_{2}^{o}$ & 0.148 & 0.018 & 0.016 \\
$3 \mathrm{p}^{4}{ }^{3} \mathrm{P}_{2}$ & $3 \mathrm{p}^{3}\left({ }^{2} \mathrm{D}\right) 3 \mathrm{~d}{ }^{3} \mathrm{P}_{2}^{o}$ & 0.006 & 0.792 & 0.771 \\
$3 \mathrm{p}^{4}{ }^{3} \mathrm{P}_{2}$ & $3 \mathrm{p}^{3}\left({ }^{2} \mathrm{D}\right) 3 \mathrm{~d}{ }^{3} \mathrm{P}_{1}^{o}$ & $6.7-4$ & 0.183 & 0.161 \\
$3 \mathrm{p}^{4}{ }^{3} \mathrm{P}_{2}$ & $3 \mathrm{p}^{3}\left({ }^{4} \mathrm{~S}\right) 3 \mathrm{~d}{ }^{3} \mathrm{D}_{3}^{o}$ & 0.589 & 1.480 & 1.490 \\
$3 \mathrm{p}^{4}{ }^{3} \mathrm{P}_{2}$ & $3 \mathrm{p}^{3}\left({ }^{4} \mathrm{~S}\right) 3 \mathrm{~d}{ }^{3} \mathrm{D}_{2}^{o}$ & 0.106 & 0.231 & 0.238 \\
$3 \mathrm{p}^{4}{ }^{3} \mathrm{P}_{1}$ & $3 \mathrm{p}^{3}\left({ }^{4} \mathrm{~S}\right) 3 \mathrm{~d}{ }^{3} \mathrm{D}_{2}^{o}$ & 0.178 & 1.360 & 1.364 \\
$3 \mathrm{p}^{4}{ }^{3} \mathrm{P}_{0}$ & $3 \mathrm{~s} 3 \mathrm{p}^{5}{ }^{3} \mathrm{P}_{1}^{o}$ & 0.102 & 0.056 & 0.052 \\
$3 \mathrm{p}^{4}{ }^{3} \mathrm{P}_{0}$ & $3 \mathrm{p}^{3}\left({ }^{2} \mathrm{D}\right) 3 \mathrm{~d}{ }^{3} \mathrm{P}_{1}^{o}$ & 0.003 & 0.831 & 0.953 \\
\hline
\end{tabular}

CIV3: earlier calculations of El-Maaref et al. [5] with the cIv3 code MCHF: earlier calculations of Froese Fischer et al. [8] with the MCHF code

GRASP: present calculations with the GRASP code
Table 2 Comparison of lifetimes $(\tau, \mathrm{s})$ for some levels of Sc VI. $\mathrm{a} \pm \mathrm{b}$ $\equiv \mathrm{a} \times 10^{ \pm \mathrm{b}}$

\begin{tabular}{llll}
\hline Level & CIV3 & MCHF & GRASP \\
\hline $3 \mathrm{p}^{3}\left({ }^{2} \mathrm{P}\right) 3 \mathrm{~d}{ }^{3} \mathrm{P}_{0}^{o}$ & $2.25-10$ & $2.50-09$ & $1.59-09$ \\
$3 \mathrm{p}^{3}\left({ }^{2} \mathrm{P}\right) 3 \mathrm{~d}{ }^{3} \mathrm{P}_{1}^{o}$ & $1.51-10$ & $1.86-09$ & $8.91-10$ \\
$3 \mathrm{p}^{3}\left({ }^{2} \mathrm{P}\right) 3 \mathrm{~d}{ }^{3} \mathrm{P}_{2}^{o}$ & $8.02-11$ & $8.66-10$ & $5.49-10$ \\
$3 \mathrm{p}^{3}\left({ }^{2} \mathrm{D}\right) 3 \mathrm{~d}{ }^{3} \mathrm{P}_{2}^{o}$ & $1.71-09$ & $1.28-11$ & $1.25-11$ \\
$3 \mathrm{p}^{3}\left({ }^{2} \mathrm{D}\right) 3 \mathrm{~d}^{3} \mathrm{P}_{1}^{o}$ & $5.12-09$ & $1.29-11$ & $1.26-11$ \\
$3 \mathrm{p}^{3}\left({ }^{2} \mathrm{D}\right) 3 \mathrm{~d}{ }^{3} \mathrm{P}_{0}^{o}$ & $1.05-08$ & $1.25-11$ & $1.22-11$ \\
\hline
\end{tabular}

CIV3: earlier calculations of El-Maaref et al. [5] with the cIv3 code MCHF: earlier calculations of Froese Fischer et al. [8] with the MCHF code

GRASP: present calculations with the GRASP code

Agreement between the GRASP and MCHF calculations is generally highly satisfactory and is within a factor of two. These differences are understandable, because $f$-values for most of these levels are rather small in magnitude. However, discrepancies with the corresponding CIV3 results of El-Maaref et al. [5] are indeed very large, and have arisen because of the corresponding differences in the $f$ - (and $A$-) values, noted in Sect. 3.

\section{Conclusions}

In a recent paper, El-Maaref et al. [5] have reported results for energy levels, $f$-values, $A$-values, and $\tau$ for the levels/transitions of S-like Sc VI. Most of their results are based on the CIV3 calculations. While their calculated energies are (nearly) acceptable for most levels, the corresponding results for other parameters are not, because these differ from the earlier available as well as our present work for several transitions, by up to three orders of magnitude. The main reason for such large discrepancies is the inclusion of limited (and arbitrary) CI in their work. Much more accurate, and larger, calculations already exist in the literature, which are by Froese Fischer et al. [8] and Massacrier and Artru [4], respectively. However, there is still scope for further improvement as well as extension over the existing results. This is because levels of Froese Fischer et al. are confined to those of the $3 s^{2} 3 p^{4}, 3 s 3 p^{5}$ and $3 s^{2} 3 p^{3} 3 d$ configurations only. Massacrier and Artru, on the other hand, have included considerably larger CI (among 51 configurations) and have reported data for 1889 levels, but their results for $A$-values are confined to the E1 transitions alone, whereas those with other types (E2, M1 and M2) are also desirable in the modelling of plasmas, and particularly in the determination of lifetimes. Therefore, in a recent paper [10], we have reported a complete set of data 
for all levels and their transitions, not only for S-like Sc VI, but also for V VIII, Cr IX, and Mn X

With the easy access to atomic structure (and scattering) codes, it has become (rather) straightforward to generate atomic data for various parameters. However, such data may not be of any use and may often be confusing and misleading, because generating accurate and reliable data, which can be confidently applied for modelling or diagnostics of plasmas, is still difficult to produce as several checks and comparisons are required, particularly when the earlier available results are in paucity. This is the main reason that large discrepancies, of orders of magnitude, are often observed in the reported data (for any ion), as noted here for Sc VI and highlighted earlier for many others in our earlier publications $[11,12]$. Finally, for the benefit of the readers, we will like to note that the earlier reported results by El-Maaref and co-workers are in large errors for other ions also, as discussed and explained by us for S-like Mn X [13], Kr-like W XXXIX [14] and Zn-like W XLV [15].

Open Access This article is licensed under a Creative Commons Attribution 4.0 International License, which permits use, sharing, adaptation, distribution and reproduction in any medium or format, as long as you give appropriate credit to the original author(s) and the source, provide a link to the Creative Commons licence, and indicate if changes were made. The images or other third party material in this article are included in the article's Creative Commons licence, unless indicated otherwise in a credit line to the material. If material is not included in the article's Creative Commons licence and your intended use is not permitted by statutory regulation or exceeds the permitted use, you will need to obtain permission directly from the copyright holder. To view a copy of this licence, visit http://creativecomm ons.org/licenses/by/4.0/.

\section{References}

[1] K M Aggarwal Atoms 625 (2018)

[2] M H L Pryce Astrophys. J. 1401192 (1964)

[3] F LeBlanc and G Alecian Astron. Astrophys. 477243 (2008)

[4] G Massacrier and M -C Artru Astron. Astrophys. 538 A52 (2012)

[5] A A El-Maaref, M M Abou Halaka and Y B Saddeek Indian J. Phys. 911029 (2017)

[6] A Hibbert Comput. Phys. Commun. 9141 (1975)

[7] R D Cowan. The Theory of Atomic Structure and Spectra, Univ. of Calif. Press, Berkeley, Ca, USA, 1981

[8] C Froese Fischer, G Tachiev and A Irimia At. Data Nucl. Data Tables 92607 (2006)

[9] I P Grant, B J McKenzie, P H Norrington, D F Mayers and N C Pyper Comput. Phys. Commun. 21207 (1980)

[10] K M Aggarwal At. Data Nucl. Data Tables 131101284 (2020)

[11] K M Aggarwal and F P Keenan Fusion Sci. Tech. 63363 (2013)

[12] K M Aggarwal Atoms 537 (2017)

[13] K M Aggarwal J. Elect. Spectrsc. Related Phen. 23546 (2019)

[14] K M Aggarwal (2019), available at arXiv:1909.03774.

[15] K M Aggarwal J. Quant. Spectrsc. Radiat. Transf. 231136 (2019)

Publisher's Note Springer Nature remains neutral with regard to jurisdictional claims in published maps and institutional affiliations. 